\title{
The Development of Multi-planetary Weight Measurement System for Computational Astronomy
}

\author{
Justus Chukwunonyerem ${ }^{1, ~ *}$, Ezechi Nwachukwu Emmanuel ${ }^{1}$, Daniyan Omoniyi Lanre ${ }^{1}$, \\ Onubi Peter Offor ${ }^{1}$, Donatus Nwagbara ${ }^{1}$, Ayogu Harrison Ejiofor ${ }^{1}$, Bonaventure Okere ${ }^{1}$, \\ Aliyu Nasiru', Chigozi Bethel Wali ${ }^{2}$, Nnadi Joseph Odo ${ }^{3}$, Chinedu Emeka Omulu', \\ Ogochukwu Edith Eze ${ }^{1}$, Kevin Eze Chukwunyere', Ayogu Jideofor Uchenna ${ }^{1}$, \\ Macdenis Onyekachi Egbuhuzor ${ }^{4}$ \\ ${ }^{1}$ NASRDA-Center for Basic Space Science, Nsukka, Enugu, Nigeria \\ ${ }^{2}$ NASRDA-Advanced Space Technology Applications Laboratory, Uyo, Nigeria \\ ${ }^{3}$ National Center for Equipment Maintenance and Development, University of Nigeria, Nsukka, Enugu, Nigeria \\ ${ }^{4}$ Department of Materials \& Metallurgical Engineering, University of Nigeria, Nsukka, Nigeria
}

Email address:

chukwujus@gmail.com (J. Chukwunonyerem)

${ }^{*}$ Corresponding author

\section{To cite this article:}

Justus Chukwunonyerem, Ezechi Nwachukwu Emmanuel, Daniyan Omoniyi Lanre, Onubi Peter Offor, Donatus Nwagbara, Ayogu Harrison Ejiofor, Bonaventure Okere, Aliyu Nasiru, Chigozi Bethel Wali, Nnadi Joseph Odo, Chinedu Emeka Omulu, Ogochukwu Edith Eze, Kevin Eze Chukwunyere, Ayogu Jideofor Uchenna, Macdenis Onyekachi Egbuhuzor. The Development of Multi-planetary Weight Measurement System for Computational Astronomy. American Journal of Astronomy and Astrophysics. Vol. 8, No. 4, 2020, pp. 75-79.

doi: $10.11648 /$ j.ajaa.20200804.12

Received: October 27, 2020; Accepted: November 7, 2020; Published: November 16, 2020

\begin{abstract}
In this work, the design of a multi-planetary weight measurement device comprising of a hardware and software system, capable of measuring and displaying the weight of objects in different planets of our solar system is presented. It is a microcontroller-based device indigenously designed for computational astronomy needs in multi-planetary weight measurement. It is also a fascinating basic space science tool for space science enthusiasts. The methodology of the hardware design involved the programming and interconnection of modular electronic chips and sensors such as load cell sensorTAS606, load cell amplifier-HX711, liquid crystal display-LCD, Joy-Stick switch and microcontroller which can measure weight of objects not exceeding five kilograms. The software system was designed into executable program using python programing language and can measure any range of object's weight in the solar system. Surface gravity values for each planet were calculated and the respective values mapped for each select planet. Weight computational codes for respective planets were also developed in C-programming language for determining the weight of different objects on each planet. The device was calibrated using a known weight of one Kilogram $(1 \mathrm{Kg})$. Accurate weights of two different objects were measured for different planets on the system; the weights (data) measured were recorded and analyzed to show the key importance of gravity in astronomical weight computation in different planets.
\end{abstract}

Keywords: Multi-Planetary, Space Science, Astronomy, Weight, Measurement, Sensors

\section{Introduction}

This work is designed and presented to demonstrate weight measurement in different planets of the solar system. It is an indigenous design comprising of a hardware device and a software program developed to drive down the concept of astronomy computation in multi-planetary weight measurement. From the works of Sir Isaac Newton, weight of an object has been defined as the product of mass of the object and the object's force of acceleration due to gravity [1]. Mass of any object is the total body matter content of the object. It is measured in grams and has the same value irrespective of the position of the object in any planet of the 
solar system [1, 2]. Weight is a vector quantity, the gravitational force which is a component of weight, has direction, it is measured in Newton and its value depends on the position of the object in any of the planets of the solar system [3]. There are eight known planets in the solar system comprising of Mecury, Venus, Earth, Mars, Jupitar, Saturn, Uranus and Neptune [4]. These planets have different surface gravity values. A stone of mass 20 grams will experience different force of gravity acting on it when placed in different planets of the solar system. The different force of gravity acting on the stone in different planets will account for different weights of the stone when measured on the planets respectively. The different values of the gravitational force in the planets also account for how objects will fall when released from each of the planets of the solar system.

This project utilized different surface gravity values for each planet $[5,6,7]$; to calculate the weight (product of object's mass and surface gravity) of an object in respective planets.

The different surface gravity values used in this work was gotten from Planetary Fact Sheet [8] and the National School Observatory - NSO websites; [9].

The major objective of this project is to demonstrate the concept of astronomical weight measurement of objects in different planets of our solar system using indigenously designed stand-alone hardware and executable software system. It is an indigenous design comprising of a hardware device and an executable software system developed to drive down the concept of astronomy computation in multiplanetary weight measurement.

It is also used for educational purpose for school children. It teaches them why a particular object would have different weights on the surface of the moon and on different planets in our solar system. It also teaches students the concept of weightlessness in space and why Astronauts float like a piece of feather in the space ship at the International space station.
This project was embarked upon to demonstrate the indigenous capability of the National Space Research and Development Agency-Center for Basic Space Science (NASRDA-CBSS), Nsukka, Enugu State Nigeria, in the design of space related spin-offs meant for educational purpose, capable of exciting young minds and space enthusiast to pursue STEM related subjects. It is also in support of the mandate of NASRDA-CBSS in providing basic space science instrument needs of the immediate society.

\section{Methodology of the Design}

The methodology of the design for the hardware involved the programming and interconnection of modular electronic chips and sensors used to realize the hardware device. These include; load cell sensor-TAS606, load cell amplifier-HX711, liquid crystal display-LCD, Joy-Stick switch and microcontroller which are found very useful in embedded system designs $[10,11]$. The hardware device can measure the weight of objects not exceeding five kilograms, a design choice made by using a load cell sensor of $5 \mathrm{~kg}$ capacity. This is to ensure the load cell is not deformed by excessive load [12, 13]. The programming code for the hardware device was written in C-programming language, the acceptable program for the microcontroller. The different Surface gravity values for each planet, gotten from the Planetary Fact Sheet and planet datasheet of the National School Observatory - NSO website were mapped for each select planet on the joy-stick switch. Weight computational codes for respective planets were developed in C-programming language for determining the weight of different objects on each planet. The hardware device was calibrated using a known weight of one Kilogram $(1 \mathrm{Kg})$, this is to ensure accurate measurement of the weight of any object when placed on the hardware device, irrespective of the planet where the object weight is measured [14].

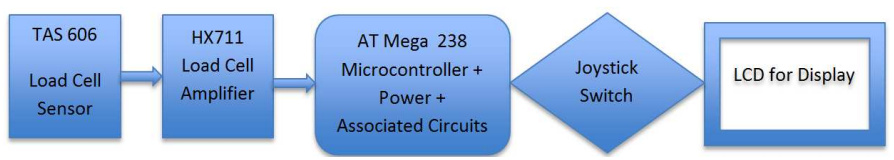

Figure 1. Block Diagram of the Hardware Design.

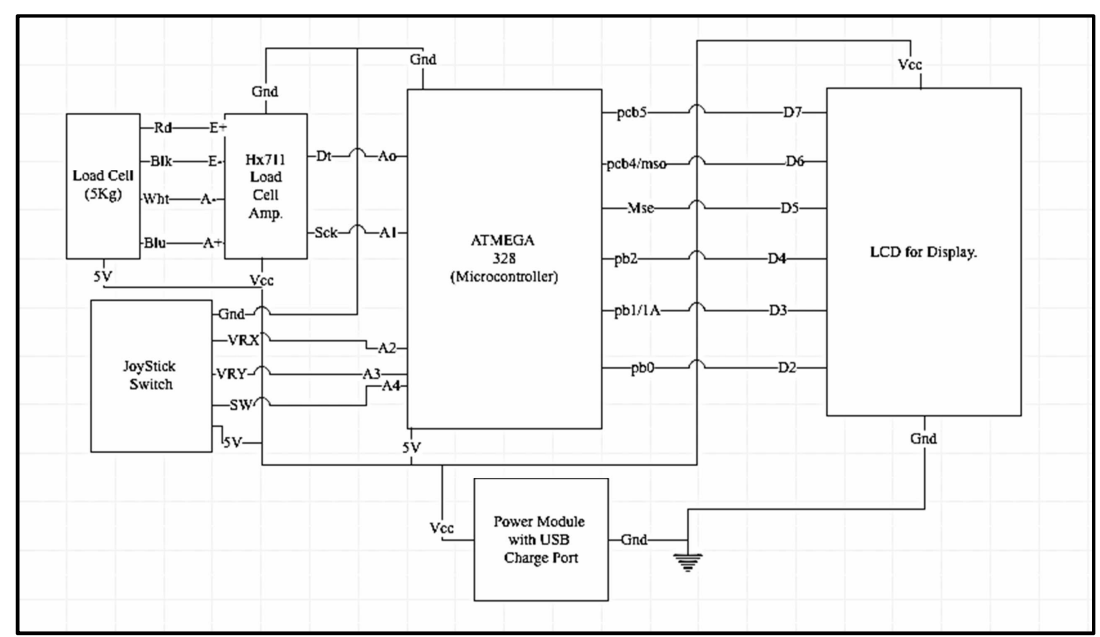

Figure 2. The Circuit Diagram of the Hardware System. 
The circuit diagram of the hardware system was designed using electronic circuit diagram software, this is as shown in the figure 2 above.

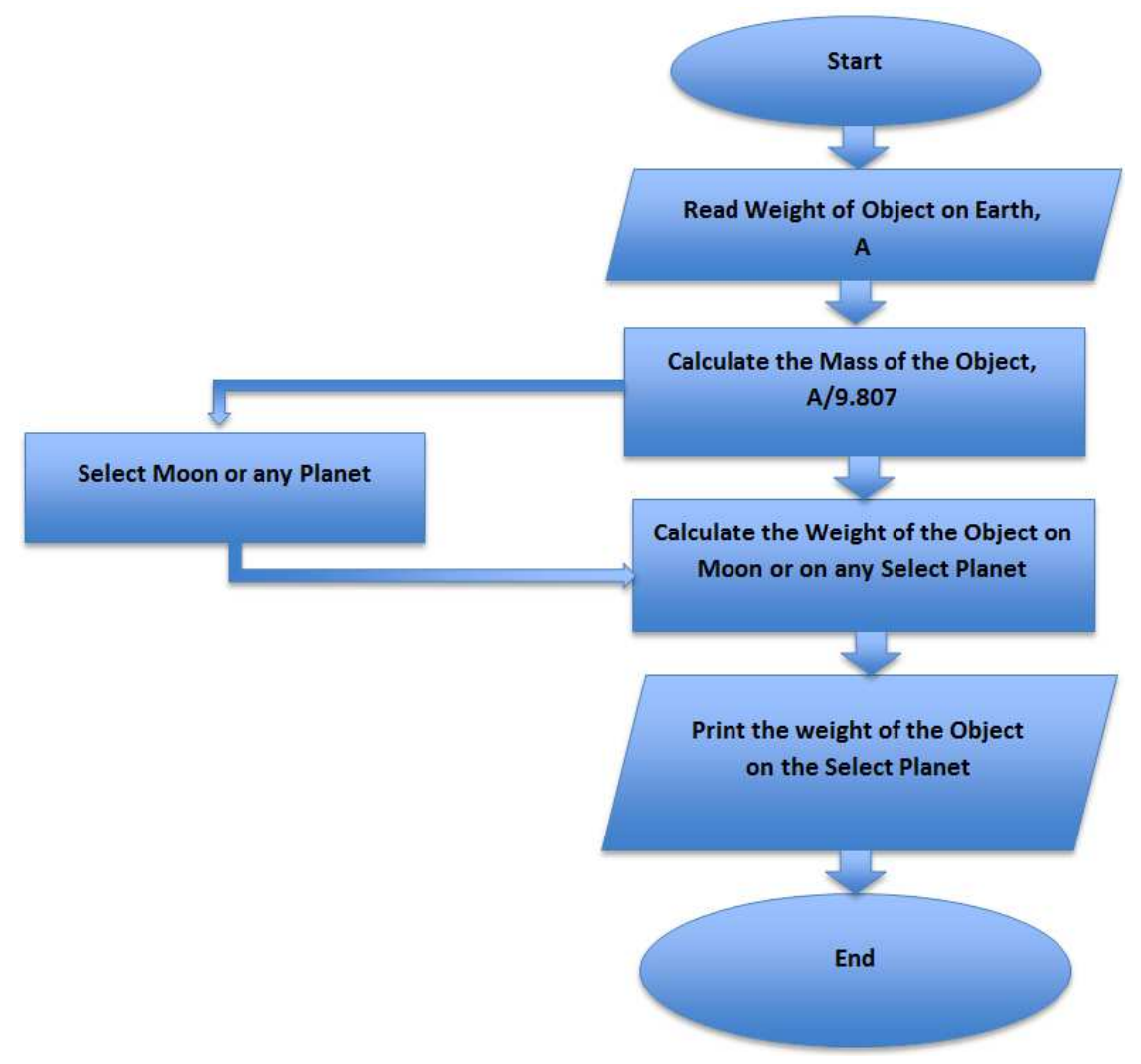

Figure 3. Flowchart of the Weight Comparator Software.

The multi-planetary weight comparator software system was designed into executable program using python programing language [15] and can measure any range of object's weight in the solar system. It was designed using the python Graphical User Interface (GUI) module - Tkinter with three interfaces. At the first run, it shows an interface that displays the name of the software - Multi-planetary Weight Comparator. It has the image of the sun and all the planets in the solar system, all shown to scale, a text that ask the user, "Ever imagined why Astronauts float in space like a piece of feather? Check out what weights looks like out there", and a call to action button named "Click". The Click button creates the second interface, where the user enters in the input field provided, the value of weight of an object on earth, which the user wants to know what it will weigh on the surface of the moon and on the surface of all the other planets in our solar system. The call to action button "CALCULATE" displays the corresponding value of what the object would weigh on Moon and all the planets in our solar system.

The second interface also has the image of the Moon and all the planets in our solar system displayed. Underneath each image, is a call to action button. This button displays key information about the moon or any of the eight known planets of the solar system when any of the planets or the moon is highlighted.

The "Want to Know More" call to action button on the second interface takes the user to the third interface. On the third interface, is an explanation telling the user the reason why a particular object will have different weight on different planets. Figure 2 above shows the flowchart of the software design.

\section{Results and Evaluation}

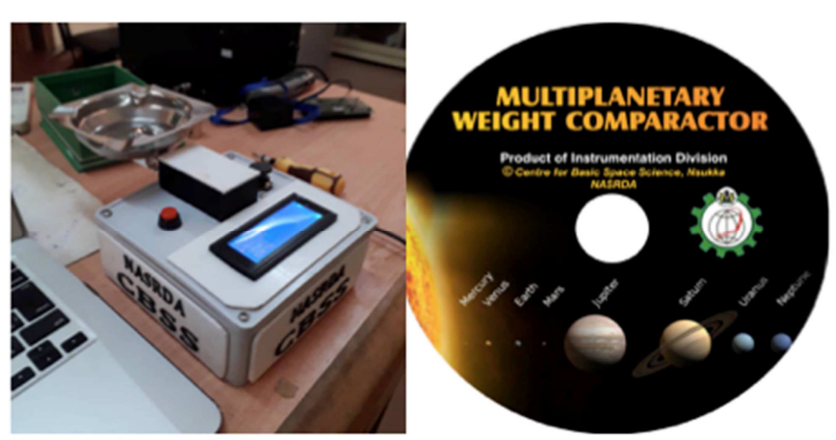

Figure 4. The designed hardware and the software system.

Figure 4 above shows the designed system comprising of the hardware and the software. Using standard known weight blocks of 500 grams and 750 grams, the hardware system was tested. The result produced accurate astronomical weight measurements of the objects on earth, moon and in different planets of the solar system. 
Table 1. Values of known object weights measured on the system.

\begin{tabular}{|c|c|c|c|}
\hline \multicolumn{2}{|c|}{ Standard Known Object Weight in Grams. } & \multirow{2}{*}{$\begin{array}{l}500 \text { Grams } \\
\text { Measured Weight of Known Object (500 } \\
\text { Grams) on the System in Grams. }\end{array}$} & \multirow{2}{*}{$\begin{array}{l}750 \text { Grams } \\
\text { Measured Weight of Known Object } \\
\text { (750 Grams) on the System in Grams. }\end{array}$} \\
\hline $\begin{array}{l}\text { Planets of the Solar } \\
\text { System: }\end{array}$ & $\begin{array}{l}\text { Surface Gravity Values for } \\
\text { different Planets and Moon: }\end{array}$ & & \\
\hline Mercury & 0.378 & 189.000 & 283.500 \\
\hline Venus & 0.907 & 453.500 & 680.250 \\
\hline Earth & 1.000 & 500.000 & 750.000 \\
\hline Mars & 0.377 & 188.500 & 282.750 \\
\hline Jupiter & 2.360 & 1180.000 & 1770.000 \\
\hline Saturn & 0.916 & 458.000 & 687.000 \\
\hline Uranus & 0.889 & 444.500 & 666.750 \\
\hline Neptune & 1.120 & 560.000 & 840.000 \\
\hline Moon (Not a Planet) & 0.166 & 83.000 & 124.500 \\
\hline
\end{tabular}

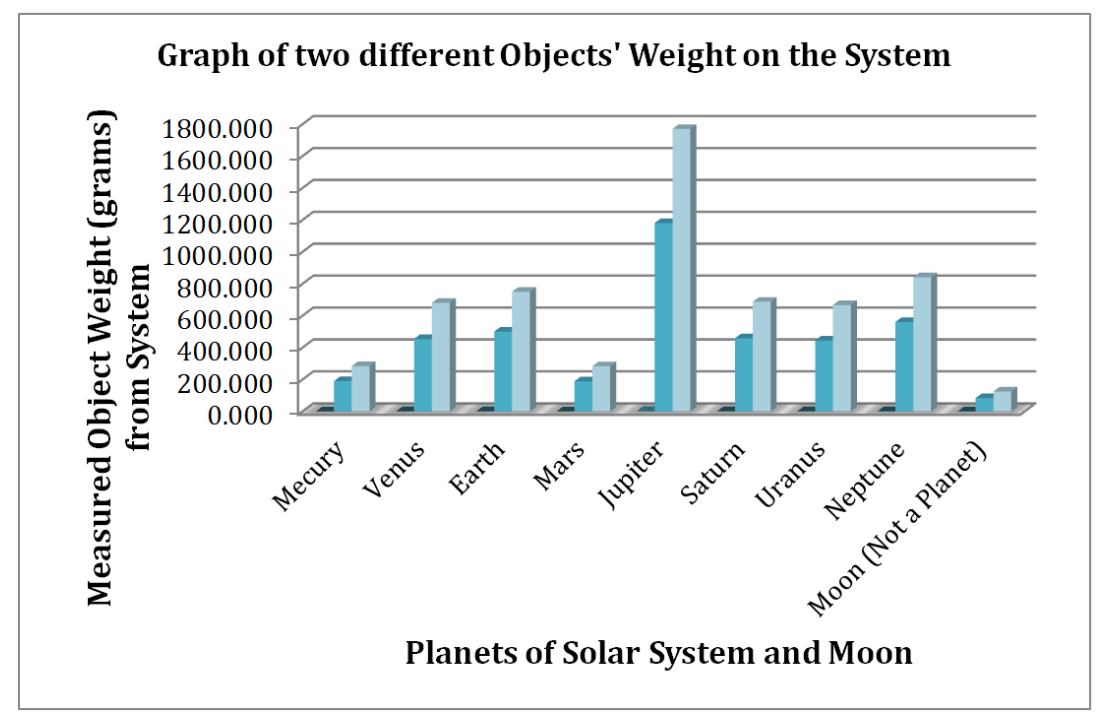

Figure 5. Graph of weights of two different objects measured on the system.

The table 1 above shows the different weight measurements generated on the system for moon and other different planets of our solar system, these results took into consideration the different surface gravity values used for different planets in the developed parent code for weight computation on the system. From table 1, a graph of two different weights measured on the system was plotted using the Pandas module for data analytics in python. This graph is presented in figure 5 above. The result on the graph shows that the weights of the objects were highest on the planet Jupiter with the highest surface gravity value, while weights of the objects were least on moon with the least surface gravity value. This goes further to validate that gravity plays very important role in astronomical weight computation in the different planets of our solar system.

\section{Conclusion}

The development of multi-planetary measurement system comprising of a software and hardware systems has been presented. The system hardware and software program was indigenously designed using microcontroller based devices, sensors, python programming language and surface gravity value, which is unique for each planet and the moon. The system was used to demonstrate astronomical weight computation in different planets of the solar system. Using two objects of known weights, validation and testing was carried out on the system. The measured weights (data) of these two objects on the system were recorded and analyzed for different planets and the moon. A graph of the recorded data was generated and analyzed using the Pandas module for data analysis in python. The graph showed that the weights of these objects are highest in planet with the highest surface gravity value, while the weights of these same objects are lowest in planet with the smallest surface gravity value. This goes further to validate the important role which gravity plays for weight computation in different planets of the solar system.

\section{References}

[1] Lawrence, J. L. (2019). Celestial Calculations: A Gentle Introduction to Computational Astronomy. MIT Press.

[2] Slater, T. F. (2020). Celestial Calculations: A Gentle Introduction to Computational Astronomy. $\mathrm{AmJPh}, 88$ (1), 8181. DOI: $10.1119 / 10.0000284$. 
[3] Joos, G., \& Freeman, I. M. (2013). Theoretical physics. Courier Corporation.

[4] Murray, C. D., \& Dermott, S. F. (1999). Solar system dynamics. Cambridge university press.

[5] Southworth, J., Wheatley, P. J., \& Sams, G. (2007). A method for the direct determination of the surface gravities of transiting extrasolar planets. Monthly Notices of the Royal Astronomical Society: Letters, 379 (1), L11-L15.

[6] Newcomb, S. (1895). On the principal fundamental constants of astronomy. The Astronomical Journal, 14, 185-189.

[7] Kuchynka, P., \& Folkner, W. M. (2013). A new approach to determining asteroid masses from planetary range measurements. Icarus, 222 (1), 243-253.

[8] NASA, (2020). NASA Space Science Coordinated Data Archive. Retrieved 8 August 2020, from https://www.nssdc.gsfc.nasa.gov.

[9] National Schools' Observatory, (2020). Planet Data Sheet. Retrieved 9 August 2020, from https://www.schoolsobservatory.org/discover/quick/weight.
[10] Wilmshurst, T. (2006). Designing embedded systems with PIC microcontrollers: principles and applications. Elsevier.

[11] Gridling, G., \& Weiss, B. (2007). Introduction to microcontrollers. Vienna University of Technology Institute of Computer Engineering Embedded Computing Systems Group.

[12] Kheiralla, A. F., Abbas Eltigani, M. M., Elzaki, A. O., \& Hamed, M. (2012). Design and Development and Calibration ofan On-board Weighing System for an Industrial Wheel Loader. In International Conference on Trends in Industrial and Mechanical Engineering (ICTIME'2012).

[13] Lin, T. T., \& Chen, W. T. (2017). Plant growth weight measurement instrument and method thereof U.S. Patent No. 9,775,300. Washington, DC: U.S. Patent and Trademark Office.

[14] Montagnino, J. G., \& Biang, J. M. (2003). Planetary weigh scale. U.S. Patent No. 6,608,260. Washington, DC: U.S. Patent and Trademark Office.

[15] Hunt, J. (2019). Advanced Guide to Python 3 Programming. Springer. 